\title{
Young's inequality in trace-class operators
}

\section{Martín Argerami • Douglas R. Farenick}

Received: 20 December 2001 / Revised version: 11 July 2002 /

Published online: 10 February 2003 - @ S Springer-Verlag 2003

Abstract. If $a$ and $b$ are trace-class operators, and if $u$ is a partial isometry, then $\left\|u\left|a b^{*}\right| u^{*}\right\|_{1} \leq$ $\left\|\frac{1}{p}|a|^{p}+\frac{1}{q}|b|^{q}\right\|_{1}$, where $\|\cdot\|_{1}$ denotes the norm in the trace class. The present paper characterises the cases of equality in this Young inequality, and the characterisation is examined in the context of both the operator and the Hilbert-Schmidt forms of Young's inequality.

Mathematics Subject Classification (2000): 47A63, 15A60

\section{Introduction}

Building on the work by T. Ando [A], it was shown in [EFZ] that for each pair of compact operators $a$ and $b$, and for each $p, q \in \mathbb{R}^{+}$for which $1 / p+1 / q=1$, there exists a partial isometry $u$ such that $u^{*} u=1-\left[\operatorname{ker}\left|a b^{*}\right|\right]$ (where $\left[\operatorname{ker}\left|a b^{*}\right|\right]$ denotes the projection onto $\left.\operatorname{ker}\left|a b^{*}\right|\right)$ and

$$
u\left|a b^{*}\right| u^{*} \leq \frac{1}{p}|a|^{p}+\frac{1}{q}|b|^{q} .
$$

The case $p=q=2$ is the arithmetic-geometric mean inequality, which was first uncovered in [BK]. In finite dimensions, the operator $u$ in (1.1) can always be required to be a unitary.

The problem of characterising the cases of equality in the operator Young inequality (1.1) was settled by O. Hirzallah and F. Kittaneh in [HK] for HilbertSchmidt operators. A very surprising feature of Hirzallah and Kittaneh's proof is that the analysis of the cases of equality in the operator inequality (1.1) occurs through a much weaker assumption. Specifically, the fact that the operators $u\left|a b^{*}\right| u^{*}$ and $\frac{1}{p}|a|^{p}+\frac{1}{p}|b|^{q}$ are equal has no role in the proof; rather, one need only know that the Hilbert-Schmidt norms of the operators $u\left|a b^{*}\right| u^{*}$ and $\frac{1}{p}|a|^{p}+\frac{1}{p}|b|^{q}$ are equal.

\section{ARGERAMI}

Departamento de Matemática, Facultad de Ciencias Exactas, Universidad Nacional de La Plata, CC 172 (1900) La Plata, Argentina (e-mail: martin@mate. unlp. edu .ar)

D. R. FARENICK

Department of Mathematics and Statistics, University of Regina, Regina, Saskatchewan S4S 0A2, Canada (e-mail: farenick@math.uregina.ca) 
In this paper we prove that for trace-class operators the Hilbert-Schmidt condition can replaced by equality in trace norm (Theorems 2.1 and 2.2). Moreover, in Theorem 4.1 we show that

$$
\left\|u\left|a b^{*}\right| u^{*}\right\|_{1}=\left\|\frac{1}{p}|a|^{p}+\frac{1}{q}|b|^{q}\right\|_{1}
$$

if and only if

$$
\left\|u\left|a b^{*}\right| u^{*}\right\|_{2}=\left\|\frac{1}{p}|a|^{p}+\frac{1}{q}|b|^{q}\right\|_{2},
$$

but neither of these equalities imply operator equality in (1.1).

Motivated by the operator Young inequality (1.1), our results incorporate the use of partial isometries as an added feature. Our computations rely on a careful analysis of the eigenvalues of the operators, and also on some convexity arguments about perturbations by unitaries.

We now introduce some notation and conventions. Suppose that $\mathcal{H}$ is a complex, separable Hilbert space and that $B(\mathcal{H})$ is the algebra of bounded linear operators acting on $\mathcal{H}$. For $x \in B(\mathcal{H})$, we denote by $R[x]$ the projection of $\mathcal{H}$ onto the closed range $\overline{\operatorname{ran} x}$ of $x$. For any operator $z,|z|$ shall denote $\left(z^{*} z\right)^{1 / 2}$, the positive square root of $z^{*} z$. If $z \in B(\mathcal{H})$ is compact, then the nonnegative real number $s_{k}(z)$, for every $k \in \mathbb{Z}^{+}$, denotes the $k$-th singular value of $z$, namely

$$
s_{k}(z)=\lambda_{k}(|z|),
$$

where

$$
\begin{aligned}
\lambda_{k}(|z|)= & \min \left\{\max \left\{\langle|z| \xi, \xi\rangle: \xi \in M^{\perp},\|\xi\|=1\right\}:\right. \\
& M \subset H, \operatorname{dim} M=k-1\} .
\end{aligned}
$$

Trace-class and Hilbert-Schmidt operators are defined via the sequence of singular values. An operator $x$ is of trace class if $\left\{s_{k}(x)\right\}_{k \in \mathbb{Z}^{+}} \in \ell^{1}\left(\mathbb{Z}^{+}\right)$, and $x$ is a Hilbert-Schmidt operator if $\left\{s_{k}(x)\right\}_{k \in \mathbb{Z}^{+}} \in \ell^{2}\left(\mathbb{Z}^{+}\right)$. Thus, the trace norm $\|\cdot\|_{1}$ and Hilbert-Schmidt norm $\|\cdot\|_{2}$ on the ideals of trace-class operators and Hilbert-Schmidt operators, respectively, are:

$$
\|x\|_{1}=\sum_{k=1}^{\infty} s_{k}(x)
$$

and

$$
\|x\|_{2}=\left(\sum_{k=1}^{\infty} s_{k}(x)^{2}\right)^{1 / 2} .
$$

The trace $\operatorname{tr}(x)$ of a trace-class operator $x$ is defined to be

$$
\operatorname{tr}(x)=\sum_{k=1}^{\infty}\left\langle x \phi_{k}, \phi_{k}\right\rangle,
$$


where $\left\{\phi_{k}\right\}_{k \in \mathbb{Z}^{+}}$is any orthonormal basis of $\mathcal{H}$. It is well known that the trace is independent of the choice of orthonormal basis and that $\operatorname{tr}(x g)=\operatorname{tr}(g x)$ if $x \in B(\mathcal{H})$ is of trace class and $g \in B(\mathcal{H})$ is arbitrary.

If $z$ is a positive compact operator, then the singular values of $z$ are simply the eigenvalues of $z$ and the list $\left\{\lambda_{k}(z)\right\}_{k \in \mathbb{Z}^{+}}$accounts for all the nonzero eigenvalues of $z$ (of which there may be finitely or infinitely many). Hence, equations (1.2) and (1.4) become, for positive trace-class operators $z$,

$$
\|z\|_{1}=\operatorname{tr} z=\sum_{k=1}^{\infty} \lambda_{k}(z) .
$$

Finally, we will also make use of some basic facts from the theory of majorisation, for which our main reference is the book of Bhatia [Ba].

\section{Equality in Young's inequality}

We begin by mentioning the trivial fact that if $p, q \in \mathbb{R}^{+}$and $1 / p+1 / q=1$, then $p>1, q>1$. Thus, if $a, b \in B(\mathcal{H})$ are positive trace-class operators, then both $a^{p}$ and $b^{q}$ are positive trace-class operators.

The following theorem is the main result of the present paper.

Theorem 2.1. Let $a, b \in B(\mathcal{H})$ be positive trace-class operators, and suppose that $u \in B(\mathcal{H})$ is a partial isometry. Assume that $p, q \in \mathbb{R}^{+}$are such that $\frac{1}{p}+\frac{1}{q}=1$. Then

$$
\operatorname{tr}\left(u|a b| u^{*}\right)=\operatorname{tr}\left(\frac{1}{p} a^{p}+\frac{1}{q} b^{q}\right)
$$

if and only if

$$
b^{q}=a^{p}, \quad \text { and } \quad R\left[u^{*}\right] \geq R[b] .
$$

Proof. Assume first that $b^{q}=a^{p}$, and $R\left[u^{*}\right] \geq R[b]$. This last condition can be stated as $u^{*} u b=b$. Note that

$$
a b=b^{q / p+1}=b^{q} .
$$

Then,

$$
\begin{aligned}
\operatorname{tr}\left(u|a b| u^{*}\right) & =\operatorname{tr}\left(u\left|b^{q}\right| u^{*}\right)=\operatorname{tr}\left(u b^{q} u^{*}\right) \\
& =\operatorname{tr}\left(u^{*} u b^{q}\right)=\operatorname{tr}\left(b^{q}\right) \\
& =\operatorname{tr}\left(\frac{1}{p} a^{p}+\frac{1}{q} b^{q}\right),
\end{aligned}
$$

which proves the "if" part. 
Now we assume that $a, b$ are positive trace-class operators satisfying

$$
\operatorname{tr}\left(u|a b| u^{*}\right)=\frac{1}{p} \operatorname{tr}\left(a^{p}\right)+\frac{1}{q} \operatorname{tr}\left(b^{q}\right),
$$

for some partial isometry $u$. In order to prove that $a^{p}=b^{q}$, we can assume without loss of generality that $u=1$. Indeed, since $u$ is a partial isometry we have that both $u^{*} u$ and $u u^{*}$ are projections, so

$$
\begin{aligned}
\frac{1}{p} \operatorname{tr}\left(a^{p}\right)+\frac{1}{q} \operatorname{tr}\left(b^{q}\right) & =\operatorname{tr}\left(u|a b| u^{*}\right)=\operatorname{tr}\left(|a b|^{1 / 2} u^{*} u|a b|^{1 / 2}\right) \\
& \leq \operatorname{tr}(|a b|) \leq \frac{1}{p} \operatorname{tr}\left(a^{p}\right)+\frac{1}{q} \operatorname{tr}\left(b^{q}\right),
\end{aligned}
$$

the last inequality being derived from Proposition 3.3 of [EFZ] (Young's inequality in eigenvalues). Then, equations (2.1) and (2.2) imply

$$
\operatorname{tr}(|a b|)=\frac{1}{p} \operatorname{tr}\left(a^{p}\right)+\frac{1}{q} \operatorname{tr}\left(b^{q}\right) .
$$

Equation (2.3) leads to the following inequalities (noted in [Z]):

$$
\begin{aligned}
\frac{1}{p} \sum_{j=1}^{\infty} \lambda_{j}(a)^{p}+\frac{1}{q} \sum_{j=1}^{\infty} \lambda_{j}(b)^{q} & =\frac{1}{p} \sum_{j=1}^{\infty} \lambda_{j}\left(a^{p}\right)+\frac{1}{q} \sum_{j=1}^{\infty} \lambda_{j}\left(b^{q}\right) \\
(\text { using }(2.3)) & =\sum_{j=1}^{\infty} \lambda_{j}(|a b|)=\sum_{j=1}^{\infty} s_{j}(|a b|) \\
& =\sum_{j=1}^{\infty} s_{j}(a b) \\
& \leq \sum_{j=1}^{\infty} s_{j}(a) s_{j}(b)=\sum_{j=1}^{\infty} \lambda_{j}(a) \lambda_{j}(b) \\
& \leq \sum_{j=1}^{\infty}\left(\frac{1}{p} \lambda_{j}(a)^{p}+\frac{1}{q} \lambda_{j}(b)^{q}\right) \\
& =\frac{1}{p} \sum_{j=1}^{\infty} \lambda_{j}(a)^{p}+\frac{1}{q} \sum_{j=1}^{\infty} \lambda_{j}(b)^{q} .
\end{aligned}
$$


In the above we have used [GK, page 63] and the scalar Young inequality for the first and second inequalities respectively. The inequalities above imply that

$$
\sum_{j=1}^{\infty} \lambda_{j}(a) \lambda_{j}(b)=\frac{1}{p} \sum_{j=1}^{\infty} \lambda_{j}(a)^{p}+\frac{1}{q} \sum_{j=1}^{\infty} \lambda_{j}(b)^{q} .
$$

Moreover, we know for each $j$ that $\lambda_{j}(a) \lambda_{j}(b) \leq \frac{1}{p} \lambda_{j}(a)^{p}+\frac{1}{q} \lambda_{j}(b)^{q}$, and so the equality obtained above implies that $\lambda_{j}(a) \lambda_{j}(b)=\frac{1}{p} \lambda_{j}(a)^{p}+\frac{1}{q} \lambda_{j}(b)^{q}$ for every $j \in \mathbb{Z}^{+}$. Since these are cases of equality in the scalar Young inequality, we have that $\lambda_{j}(a)^{p}=\lambda_{j}(b)^{q}$ for every $j \in \mathbb{Z}^{+}$.

Now we express $\mathcal{H}$ as two direct sums:

$$
\mathcal{H}=\overline{\operatorname{ran} a} \oplus \operatorname{ker} a=\overline{\operatorname{ran} b} \oplus \operatorname{ker} b .
$$

Since the sequences $\left\{\lambda_{k}(a)\right\}_{k \in \mathbb{Z}^{+}}$and $\left\{\lambda_{k}(b)\right\}_{k \in \mathbb{Z}^{+}}$capture all the nonzero eigenvalues of $a$ and $b$, with multiplicities counted, we deduce that the Hilbert spaces $\overline{\operatorname{ran} a}$ and $\overline{\operatorname{ran} b}$ are isomorphic. Let $w_{0}$ be a unitary implementing this isomorphism; it can be viewed as a partial isometry in $B(\mathcal{H})$ with initial space $\overline{\operatorname{ran} a}$ and final space $\overline{\operatorname{ran} b}$. The computations we have done to this stage do not give us any information about the relation between $\operatorname{ker} a$ and $\operatorname{ker} b$; in particular, there is no reason why ker $a$ and $\operatorname{ker} b$ should be isomorphic. To address this difficulty, we consider new operators $\tilde{a}$ and $\tilde{b}$ on $\mathcal{H} \oplus \mathcal{H}$, namely $\tilde{a}=a \oplus 0$ and $\tilde{b}=b \oplus 0$. Notice that, if we consider $\mathcal{H}$ embedded as the "first coordinate" of $\mathcal{H} \oplus \mathcal{H}$, we have that $\operatorname{ran} \tilde{a}=\operatorname{ran} a$ and $\operatorname{ran} \tilde{b}=\operatorname{ran} b$. The benefit of this situation is that we now can say that $\operatorname{ker} \tilde{a}$ and $\operatorname{ker} \tilde{b}$ have the same (infinite) dimension, and therefore the partial isometry $w_{0}$ extends to a unitary $w$ acting on $\mathcal{H} \oplus \mathcal{H}$ such that $\tilde{b}^{q}=w \tilde{a}^{p} w^{*}$.

For these extended operators we still have equality (2.3), which becomes

$$
\operatorname{tr}\left(\left|\tilde{a} w \tilde{a}^{p / q} w^{*}\right|\right)=\operatorname{tr}\left(\tilde{a}^{p}\right)
$$

when we take into account that now $w$ is a unitary. Our aim now is to prove that the unitary $w$ commutes with $\tilde{a}$. To achieve that, it is enough, by functional calculus, to show that $w$ commutes with $\tilde{a}^{p}$. So in our formulas we can replace $\tilde{a}$ by $\tilde{a}^{1 / p}$ without loss of generality: we will prove then that $w$ commutes with $\tilde{a}^{p}$, which is equivalent to $w$ commuting with $\tilde{a}$. Therefore, the equality that we now analyse is

$$
\operatorname{tr}\left(\left|\tilde{a}^{1 / p} w \tilde{a}^{1 / q} w^{*}\right|\right)=\operatorname{tr}(\tilde{a}),
$$

where we have renamed $\tilde{a}^{p}$ as $\tilde{a}$. 
Equation (2.5) can also be written as $\operatorname{tr}\left(\left|\tilde{a}^{1 / q} w_{1} \tilde{a}^{1 / p} w_{1}^{*}\right|\right)=\operatorname{tr}(\tilde{a})$, where $w_{1}$ is the unitary $w_{1}=w^{*}$. To see why, note that if $y=\tilde{a}^{1 / p} w \tilde{a}^{1 / q}$, then

$$
\operatorname{tr}\left(\left|\tilde{a}^{1 / p} w \tilde{a}^{1 / q} w^{*}\right|\right)=\operatorname{tr}\left(\left(w y^{*} y w^{*}\right)^{1 / 2}\right)=\operatorname{tr}\left(w\left(y^{*} y\right)^{1 / 2} w^{*}\right)=\operatorname{tr}\left(\left(y^{*} y\right)^{1 / 2}\right),
$$

the middle equality being valid because $w$ is unitary. Because $\operatorname{ker}\left(y^{*} y-\lambda 1\right)$ and $\operatorname{ker}\left(y y^{*}-\lambda 1\right)$ have the same (finite) dimension for all nonzero $\lambda$, it follows that $\operatorname{tr}\left(\left(y^{*} y\right)^{1 / 2}\right)=\operatorname{tr}\left(\left(y y^{*}\right)^{1 / 2}\right)=\operatorname{tr}\left(\left(w^{*} y y^{*} w\right)^{1 / 2}\right)=\operatorname{tr}\left(\left|y^{*} w\right|\right)=$ $\operatorname{tr}\left(\left|\tilde{a}^{1 / q} w_{1} \tilde{a}^{1 / p} w_{1}^{*}\right|\right)$.

Therefore, we assume henceforth that $p \geq 2$, for otherwise we could simply exchange $q$ for $p$ and $w_{1}$ for $w$ before continuing with the arguments that now follow.

The space $\mathcal{H} \oplus \mathcal{H}$ has an orthonormal basis $\left\{\phi_{k}\right\}_{k \in \mathbb{Z}^{+}}$of eigenvectors of $\tilde{a}$ (infinitely many of them belonging to ker $\tilde{a}$ ). Let $\left\{d_{k}\right\}_{k \in \mathbb{Z}^{+}} \subset \mathbb{R}_{0}^{+}$be such that $\tilde{a} \phi_{k}=d_{k} \phi_{k}$ for every $k \in \mathbb{Z}^{+}$, and set $w_{i j}=\left\langle w \phi_{j}, \phi_{i}\right\rangle$, for all $i, j \in \mathbb{Z}^{+}$. Then, from $w^{*} w=w w^{*}=1$,

$$
\sum_{i=1}^{\infty}\left|w_{i j}\right|^{2}=\sum_{j=1}^{\infty}\left|w_{i j}\right|^{2}=1
$$

and, for every $j$,

$$
w^{*} \phi_{j}=\sum_{k=1}^{\infty}\left\langle w^{*} \phi_{j}, \phi_{k}\right\rangle \phi_{k}=\sum_{k=1}^{\infty} \bar{w}_{j k} \phi_{k} .
$$

Using the equations above, the terms in equation (2.5) become

$$
\operatorname{tr}(\tilde{a})=\sum_{j=1}^{\infty}\left\langle\tilde{a} \phi_{j}, \phi_{j}\right\rangle=\sum_{j=1}^{\infty} d_{j},
$$

and

$$
\begin{aligned}
\operatorname{tr}\left(\left|\tilde{a}^{1 / p} w \tilde{a}^{1 / q} w^{*}\right|\right) & =\operatorname{tr}\left(\left(w \tilde{a}^{1 / q} w^{*} \tilde{a}^{2 / p} w \tilde{a}^{1 / q} w^{*}\right)^{1 / 2}\right) \\
& =\operatorname{tr}\left(w\left(\tilde{a}^{1 / q} w^{*} \tilde{a}^{2 / p} w \tilde{a}^{1 / q}\right)^{1 / 2} w^{*}\right) \\
& =\operatorname{tr}\left(\left(\tilde{a}^{1 / q} w^{*} \tilde{a}^{2 / p} w \tilde{a}^{1 / q}\right)^{1 / 2}\right) .
\end{aligned}
$$

If $\xi \in \mathcal{H}$ is a unit vector and $x \in B(\mathcal{H})^{+}$, then

$$
\left\langle x^{1 / 2} \xi, \xi\right\rangle \leq\left\|x^{1 / 2} \xi\right\|\|\xi\|=\langle x \xi, \xi\rangle^{1 / 2}
$$


Thus, using equations (2.7), (2.8), and (2.9), we obtain from (2.5)

$$
\begin{aligned}
\sum_{j=1}^{\infty} d_{j} & =\operatorname{tr}\left(\left(\tilde{a}^{1 / q} w \tilde{a}^{2 / p} w^{*} \tilde{a}^{1 / q}\right)^{1 / 2}\right)=\sum_{j=1}^{\infty}\left\langle\left(\tilde{a}^{1 / q} w \tilde{a}^{2 / p} w^{*} \tilde{a}^{1 / q}\right)^{1 / 2} \phi_{j}, \phi_{j}\right\rangle \\
& \leq \sum_{j=1}^{\infty}\left\langle\left(\tilde{a}^{1 / q} w \tilde{a}^{2 / p} w^{*} \tilde{a}^{1 / q}\right) \phi_{j}, \phi_{j}\right\rangle^{1 / 2} \\
& =\sum_{j=1}^{\infty}\left(d_{j}^{2 / q}\left\langle w \tilde{a}^{2 / p} w^{*} \phi_{j}, \phi_{j}\right\rangle\right)^{1 / 2} \\
& =\sum_{j=1}^{\infty}\left(d_{j}^{2 / q} \sum_{k, h=1}^{\infty} \bar{w}_{j k} w_{j h}\left\langle\tilde{a}^{2 / p} \phi_{k}, \phi_{h}\right\rangle\right)^{1 / 2} \\
& =\sum_{j=1}^{\infty}\left(\sum_{k=1}^{\infty} d_{j}^{2 / q} d_{k}^{2 / p}\left|w_{j k}\right|^{2}\right)^{1 / 2}=\sum_{j=1}^{\infty} d_{j}^{1 / q}\left(\sum_{k=1}^{\infty} d_{k}^{2 / p}\left|w_{j k}\right|^{2}\right)^{1 / 2} \\
& \leq\left(\sum_{j=1}^{\infty} d_{j}\right)^{1 / q}\left[\sum_{j=1}^{\infty}\left(\sum_{k=1}^{\infty} d_{k}^{2 / p}\left|w_{j k}\right|^{2}\right)^{p / 2}\right]^{1 / p},
\end{aligned}
$$

where we have used Hölder's Inequality in the last step.

Consider now the vectors

$$
x=\left(d_{j}\right)_{j}, \quad y=\left(\sum_{k=1}^{\infty} d_{k}^{2 / p}\left|w_{j k}\right|^{2}\right)_{j} .
$$

Note that from equations (2.6), the matrix $A=\left\{\left|w_{j k}\right|^{2}\right\}_{j k}$ is doubly stochastic, so $y=A x^{2 / p}$ is majorised by $x^{2 / p}$. If we consider the vectors $x^{(n)}=\left(x_{1}^{\downarrow}, \ldots, x_{n}^{\downarrow}\right)$, $y^{(n)}=\left(y_{1}^{\downarrow}, \ldots, y_{n}^{\downarrow}\right)$, where as usual the arrow means that we are considering the entries of $x$ and $y$ in decreasing order, then $y^{(n)}$ is weakly majorised by $\left(x^{(n)}\right)^{2 / p}$. Therefore, $f\left(y^{(n)}\right)$ is weakly majorised by $f\left(x^{(n)}\right)$, for every convex monotone function $f$ (see Corollary II.3.4 of [Ba]), and in particular for the function $f(x)=x^{p / 2}$ (recall that $p \geq 2$ ). Thus,

$$
\left.\operatorname{tr}\left(y^{(n)}\right)^{p / 2}\right) \leq \operatorname{tr}\left(x^{(n)}\right) \text { for every } n .
$$

Hence,

$$
\operatorname{tr}\left(y^{p / 2}\right) \leq \operatorname{tr}(x)
$$


that is,

$$
\sum_{j=1}^{\infty}\left(\sum_{k=1}^{\infty} d_{k}^{2 / p}\left|w_{j k}\right|^{2}\right)^{p / 2} \leq \sum_{j=1}^{\infty} d_{j} .
$$

Taking into account this inequality and the previous computations, we get

$$
\begin{aligned}
\sum_{j=1}^{\infty} d_{j} & \leq \sum_{j=1}^{\infty} d_{j}^{1 / q}\left(\sum_{k=1}^{\infty} d_{k}^{2 / p}\left|w_{j k}\right|^{2}\right)^{1 / 2} \\
& \leq\left(\sum_{j=1}^{\infty} d_{j}\right)^{1 / q}\left[\sum_{j=1}^{\infty}\left(\sum_{k=1}^{\infty} d_{k}^{2 / p}\left|w_{j k}\right|^{2}\right)^{p / 2}\right]^{1 / p} \\
& \leq\left(\sum_{j=1}^{\infty} d_{j}\right)^{1 / q}\left(\sum_{j=1}^{\infty} d_{j}\right)^{1 / p} \\
& =\sum_{j=1}^{\infty} d_{j} .
\end{aligned}
$$

So in particular

$$
\begin{aligned}
& \sum_{j=1}^{\infty} d_{j}^{1 / q}\left(\sum_{k=1}^{\infty} d_{k}^{2 / p}\left|w_{j k}\right|^{2}\right)^{1 / 2} \\
&=\left(\sum_{j=1}^{\infty} d_{j}\right)^{1 / q}\left[\sum_{j=1}^{\infty}\left(\sum_{k=1}^{\infty} d_{k}^{2 / p}\left|w_{j k}\right|^{2}\right)^{p / 2}\right]^{1 / p}
\end{aligned}
$$

Since this is equality in the Hölder inequality, we conclude that, for every $j$,

$$
d_{j}=\left(\sum_{k=1}^{\infty} d_{k}^{2 / p}\left|w_{j k}\right|^{2}\right)^{p / 2},
$$

which we may write as

$$
d_{j}^{2 / p}=\sum_{k=1}^{\infty} d_{k}^{2 / p}\left|w_{j k}\right|^{2} .
$$

The sequence $\left\{d_{k}\right\}_{k}$ consists of eigenvalues of $\tilde{a}$, repeated according to multiplicity. Every nonzero eigenvalue of $a$ (and hence of $\tilde{a}$ ) is repeated at most finitely many times, since $a$ is compact; the eigenvalue zero appears infinitely many times. 
Now assume that $\lambda_{1}$ is the largest positive eigenvalue of $a$, and that $m_{1}=$ $\operatorname{dim} \operatorname{ker}\left(a-\lambda_{1} 1\right)$. Then there exists a permutation $\tau$ of $\mathbb{Z}^{+}$for which $d_{\tau(k)}=\lambda_{1}$ for $k=1, \ldots, m_{1}$. Use this permutation to rewrite (2.10) as

$$
d_{\tau(j)}^{2 / p}=\sum_{k=1}^{\infty} d_{\tau(k)}^{2 / p}\left|w_{\tau(j) \tau(k)}\right|^{2}, \quad \text { for all } j \in \mathbb{Z}^{+},
$$

and to reorder the orthonormal basis of eigenvectors as $\left\{\phi_{\tau(k)}\right\}_{k}$. Thus, without loss of generality, we may assume that the sequence $\left\{d_{k}\right\}_{k}$ is already ordered in such a way that

$$
\lambda_{1}=d_{1}=d_{2}=\cdots=d_{m_{1}}>d_{k} \quad \text { for every } k \geq m_{1}+1 .
$$

Therefore, we still work with equation (2.10). For every $j=1, \ldots, m_{1}$, we have that $d_{j}^{2 / p}$ is an extremal point of the interval $\left[0, \lambda_{1}^{2 / p}\right]$. Because equation (2.10) represents, for $1 \leq j \leq m_{1}, \lambda_{1}^{2 / p}=d_{j}^{2 / p}$ as a convex combination of points from the sequence $\left\{d_{k}^{2 / p}\right\}_{k}$, equality in (2.10) can hold for each $1 \leq j \leq m_{1}$ only if

$$
\left|w_{j k}\right|^{2}=0 \quad \text { for all } k \geq m_{1}+1, \quad 1 \leq j \leq m_{1} .
$$

Thus, the matrix representation for the unitary $w$ with respect to the orthonormal basis $\left\{\phi_{k}\right\}_{k}$ has the form

$$
w=\left[\begin{array}{cc}
w_{1} & 0 \\
x & w_{2}
\end{array}\right]
$$

where $w_{1} \in M_{m_{1}}(\mathbb{C})$ is unitary. The condition $w w^{*}=1$ implies that $w_{1} x^{*}=0$, and so $x^{*}=w_{1}^{*}\left(w_{1} x^{*}\right)=0$, whence $x=0$. Therefore,

$$
w_{s t}=0, \quad \text { for every } 1 \leq t \leq m_{1}, \quad s \geq m_{1}+1,
$$

and so $\operatorname{ker}\left(\tilde{a}-\lambda_{1} 1\right)=\operatorname{Span}\left\{\phi_{1}, \ldots, \phi_{m_{1}}\right\}$ is invariant under $w$ and $w^{*}$. Thus $w p_{1}=p_{1} w$, where $p_{1}$ is the projection of $\mathcal{H} \oplus \mathcal{H}$ onto $\operatorname{ker}\left(\tilde{a}-\lambda_{1} 1\right)$.

Now consider the next largest positive eigenvalue $\lambda_{2}$ of $\tilde{a}$. Let $m_{2}=$ $\operatorname{dim} \operatorname{ker}\left(\tilde{a}-\lambda_{2} 1\right)$; then there exists a reordering of the sequence $\left\{d_{k}\right\}_{k}$ so that $\lambda_{1}=d_{k}$ for $k=1, \ldots, m_{1}$, and $\lambda_{2}=d_{k}$ for $k=m_{1}+1, \ldots, m_{1}+m_{2}$, and $\lambda_{2}>d_{j}$ if $j \geq m_{1}+m_{2}+1$. In this reordering, equation (2.10) becomes

$$
\sum_{k=1}^{m_{1}} d_{k}^{2 / p}\left|w_{j k}\right|^{2}+\sum_{k=m_{1}+1}^{\infty} d_{k}^{2 / p}\left|w_{j k}\right|^{2}=d_{j}^{2 / p},
$$

which by conditions (2.11) and (2.12) specialises to

$$
\sum_{k=m_{1}+1}^{\infty} d_{k}^{2 / p}\left|w_{j k}\right|^{2}=d_{j}^{2 / p}, \quad \text { for all } m_{1}+1 \leq j \leq m_{1}+m_{2} .
$$


Now, because $d_{m_{1}+1}^{2 / p}, \ldots, d_{m_{1}+m_{2}}^{2 / p}>d_{t}^{2 / p}$ for all $t \geq m_{1}+m_{2}+1$, the arguments that were used above prove that equation (2.13) is possible only if $\left|w_{j k}\right|=0$ for all $k \geq m_{1}+m_{2}+1$ and $m_{1}+1 \leq j \leq m_{2}$. Thus, as argued above, $\operatorname{ker}\left(\tilde{a}-\lambda_{2} 1\right)=\operatorname{Span}\left\{\phi_{m_{1}+1}, \ldots, \phi_{m_{1}+m_{2}}\right\}$ is invariant under $w$ and $w^{*}$. Hence, $w p_{2}=p_{2} w$, where $p_{2}$ is the projection of $\mathcal{H} \oplus \mathcal{H}$ onto $\operatorname{ker}\left(\tilde{a}-\lambda_{2} 1\right)$.

Now continue inductively and conclude that if $\lambda_{1}>\lambda_{2}>\cdots>0$ are the positive eigenvalues of $\tilde{a}$, and if $p_{1}, p_{2}, \ldots$ are the corresponding projections onto the eigenspaces $\operatorname{ker}\left(\tilde{a}-\lambda_{n} 1\right)$, then $w p_{n}=p_{n} w$, for each $n$. Hence, if $\|\cdot\|$ denotes the norm in $B(\mathcal{H} \oplus \mathcal{H})$, we obtain from the compactness of $\tilde{a}$ that

$$
\lim _{n}\left\|\tilde{a}-\sum_{j=1}^{n} \lambda_{j} p_{j}\right\|=0,
$$

and so

$$
\begin{aligned}
\|w \tilde{a}-\tilde{a} w\| & =\left\|w \tilde{a}-\sum_{j=1}^{n} \lambda_{j} w p_{j}+\sum_{j=1}^{n} \lambda_{j} p_{j} w-\tilde{a} w\right\| \\
& \leq\left\|w\left(\tilde{a}-\sum_{j=1}^{n} \lambda_{j} p_{j}\right)\right\|+\left\|\left(\tilde{a}-\sum_{j=1}^{n} \lambda_{j} p_{j}\right) w\right\|
\end{aligned}
$$

for every $n$, which implies that $\tilde{a} w-w \tilde{a}=0$. Therefore, $\tilde{b}^{q}=w \tilde{a}^{p} w^{*}=$ $\tilde{a}^{p} w w^{*}=\tilde{a}^{p}$. But $\tilde{a}^{p}=\tilde{b}^{q}$ if and only if $a^{p}=b^{q}$.

To finish the proof, we need to verify that $u^{*} u b=b$. We return to equation (2.1) to obtain

$$
\operatorname{tr}\left(u b^{q} u^{*}\right)=\operatorname{tr}\left(b^{q}\right)
$$

which we write as

$$
\operatorname{tr}\left(b^{q / 2} u^{*} u b^{q / 2}\right)=\operatorname{tr}\left(b^{q}\right),
$$

or equivalently as

$$
\operatorname{tr}\left(b^{q / 2}\left(1-u^{*} u\right) b^{q / 2}\right)=0 .
$$

Because $1-u^{*} u$ is a projection, we can rewrite the equation above as

$$
\operatorname{tr}\left(\left[\left(1-u^{*} u\right) b^{q / 2}\right]^{*}\left[\left(1-u^{*} u\right) b^{q / 2}\right]\right)=0 .
$$

By faithfulness of the trace we conclude that $\left(1-u^{*} u\right) b^{q / 2}=b^{q / 2}\left(1-u^{*} u\right)=0$. Thus, using functional calculus, we obtain $b u^{*} u=u^{*} u b=b$, implying that $R\left[u^{*}\right]=u^{*} u \geq R[b]$, which completes the proof.

Note that in Theorem 2.1, the condition $a^{p}=b^{q}$ implies that $R[a]=R[b]$. But this is not the case when $a, b$ are not positive, and indeed it is the projection $R[b]$ that is of interest in the extension below of Theorem 2.1 to arbitrary trace-class operators. 
Theorem 2.2. Let $a, b \in B(\mathcal{H})$ be trace-class operators and $u \in B(\mathcal{H})$ be a partial isometry. Suppose $p, q \in \mathbb{R}^{+}$are such that $\frac{1}{p}+\frac{1}{q}=1$. Then

$$
\operatorname{tr}\left(u\left|a b^{*}\right| u^{*}\right)=\operatorname{tr}\left(\frac{1}{p}|a|^{p}+\frac{1}{q}|b|^{q}\right)
$$

if and only if

$$
|b|^{q}=|a|^{p}, \quad \text { and } \quad R\left[u^{*}\right] \geq R[b] .
$$

Proof. Note that if we consider the polar decomposition $v|b|$ of $b$, then from the proof of Proposition 4.1 in [EFZ] we have

$$
\left|a b^{*}\right|=v|| a|| b|| v^{*} .
$$

Note also that $v^{*} v$ is the projection onto the range of $b^{*}$.

To prove the sufficiency, assume that $|b|^{q}=|a|^{p}$ and $R\left[u^{*}\right] \geq R[b]$. Thus, $\left(b^{*} b\right)^{q}=\left(a^{*} a\right)^{p}$ and $u^{*} u b=b$, and therefore

$$
\begin{aligned}
\operatorname{tr}\left(u\left|a b^{*}\right| u^{*}\right) & =\operatorname{tr}\left(u v|| a|| b|| v^{*} u^{*}\right)=\operatorname{tr}\left(u v|b|^{q} v^{*} u^{*}\right) \\
& =\operatorname{tr}\left(u^{*} u b|b|^{q-1} v^{*}\right)=\operatorname{tr}\left(b|b|^{q-1} v^{*}\right)=\operatorname{tr}\left(v|b|^{q} v^{*}\right) \\
& =\operatorname{tr}\left(v^{*} v|b|^{q}\right)=\operatorname{tr}\left(|b|^{q}\right) \\
& =\frac{1}{p} \operatorname{tr}\left(|a|^{p}\right)+\frac{1}{q} \operatorname{tr}\left(|b|^{q}\right) .
\end{aligned}
$$

To prove the necessity, assume that equation (2.14) holds. Using twice the same idea as in (2.2), we obtain

$$
\operatorname{tr}\left(u\left|a b^{*}\right| u^{*}\right)=\operatorname{tr}\left(u v|| a|| b|| v^{*} u^{*}\right) \leq \operatorname{tr}(|| a|| b||) .
$$

The tracial Young inequality is

$$
\operatorname{tr}(|| a|| b||) \leq \operatorname{tr}\left(\frac{1}{p}|a|^{p}+\frac{1}{q}|b|^{q}\right),
$$

(again from Proposition 3.3 of [EFZ]) and therefore

$$
\operatorname{tr}(|| a|| b||)=\operatorname{tr}\left(\frac{1}{p}|a|^{p}+\frac{1}{q}|b|^{q}\right) .
$$

Hence, by Theorem 2.1, we have that $|a|^{p}=|b|^{q}$. Therefore, the trace equation (2.14) simplifies to

$$
\operatorname{tr}\left(u v|b|^{q} v^{*} u^{*}\right)=\operatorname{tr}\left(|b|^{q}\right) .
$$


Recall that $v|b|=b$ and $|b| v^{*}=b^{*}$, and so $v|b|^{2} v^{*}=b b^{*}$. Using this,

$$
\operatorname{tr}\left(u v|b|^{q} v^{*} u^{*}\right)=\operatorname{tr}\left(u\left(b b^{*}\right)^{q / 2} u^{*}\right) .
$$

Thus, noting that $\operatorname{tr}\left(|b|^{q}\right)=\operatorname{tr}\left(\left(b b^{*}\right)^{q / 2}\right)$, equation (2.15) becomes

$$
\operatorname{tr}\left(\left(b b^{*}\right)^{q / 4}\left(1-u^{*} u\right)\left(b b^{*}\right)^{q / 4}\right)=0 .
$$

This equation and the faithfulness of the trace yield, as before,

$$
\left(1-u^{*} u\right)\left(b b^{*}\right)^{q / 4}=0 .
$$

Because $\left(b b^{*}\right)^{q / 4}$ is positive, we apply functional calculus to get $\left(1-u^{*} u\right) b b^{*}=$ 0 . Then $\left(1-u^{*} u\right) b b^{*}\left(1-u^{*} u\right)=0$, which implies that $\left(1-u^{*} u\right) b=0$; that is, $u^{*} u b=b$.

\section{Equality in the operator Young inequality}

To characterise cases of equality in the operator Young inequality ([EFZ])

$$
u\left|a b^{*}\right| u^{*} \leq \frac{1}{p}|a|^{p}+\frac{1}{q}|b|^{q},
$$

we shall employ the work in the previous section on the tracial inequality.

It is fairly easy to show that Young inequality in trace or Hilbert-Schmidt norm is strictly weaker than the operator inequality. Indeed, let $a$ be any nonzero positive trace-class operator, let $b=a^{p / q}$, and let $u$ be any unitary operator such that $u a \neq a u$. Then $u^{*} u a=a$ and

$$
\operatorname{tr}\left(u\left|a b^{*}\right| u^{*}\right)=\frac{1}{p} \operatorname{tr}\left(a^{p}\right)+\frac{1}{q} \operatorname{tr}\left(b^{q}\right)
$$

but clearly

$$
u\left|a b^{*}\right| u^{*} \neq \frac{1}{p} a^{p}+\frac{1}{q} b^{q},
$$

since the first term is $u a^{p} u^{*}$ and the second term is $a^{p}$.

Furthermore, if $a, b$ are trace-class operators and $u$ is a unitary commuting with $\left|a b^{*}\right|$, then

$$
\operatorname{tr}\left(u\left|a b^{*}\right| u^{*}\right)=\operatorname{tr}\left(\left|a b^{*}\right|\right) \leq \frac{1}{p} \operatorname{tr}\left(|a|^{p}\right)+\frac{1}{q} \operatorname{tr}\left(|b|^{q}\right),
$$

but $u\left|a b^{*}\right| u^{*}=\left|a b^{*}\right|$ can fail to be dominated as an operator by $\frac{1}{p}|a|^{p}+\frac{1}{q}|b|^{q}$, even if the Hilbert space has finite dimension.

As before, we examine the case of positive operators first. 
Theorem 3.1. Let $a, b \in B(\mathcal{H})$ be positive trace-class operators, and suppose that $u \in B(\mathcal{H})$ is a partial isometry. Let $p, q \in \mathbb{R}^{+}$such that $\frac{1}{p}+\frac{1}{q}=1$. Then the following statements are equivalent:

1. $u|a b| u^{*}=\frac{1}{p} a^{p}+\frac{1}{q} b^{q}$;

2. $b^{q}=a^{p}, R\left[u^{*}\right] \geq R[b]$, and $u b=b u$.

Proof. Write $\mathcal{H}=\mathcal{H}_{+} \oplus \mathcal{H}_{0}$, where $\mathcal{H}_{0}=\operatorname{ker} b=\operatorname{ker} b^{q}$. Thus,

$$
b=\left[\begin{array}{rr}
b_{+} & 0 \\
0 & 0
\end{array}\right] \quad \text { and } \quad u=\left[\begin{array}{ll}
u_{11} & u_{12} \\
u_{21} & u_{22}
\end{array}\right] \text {, }
$$

where $\operatorname{ker} b_{+}=\operatorname{ker} b_{+}^{q}=\{0\}$.

To prove that (1) implies (2), assume that $u|a b| u^{*}=\frac{1}{p} a^{p}+\frac{1}{q} b^{q}$. Then, of course,

$$
\operatorname{tr}\left(u|a b| u^{*}\right)=\frac{1}{p} \operatorname{tr}\left(a^{p}\right)+\frac{1}{q} \operatorname{tr}\left(b^{q}\right),
$$

which implies by Theorem 2.1 that $b^{q}=a^{p}$ and $R\left[u^{*}\right] \geq R[b]$. Thus, it remains to show that $u$ and $b$ commute.

The operator equation $u|a b| u^{*}=\frac{1}{p} a^{p}+\frac{1}{q} b^{q}$ simplifies to

$$
u b^{q} u^{*}=b^{q},
$$

which indicates that $\operatorname{ker} b^{q}=\operatorname{ker} b$ is invariant under $u^{*}$. Hence, $u_{12}=0$. Therefore, the matricial form of (3.3) is

$$
\left[\begin{array}{cc}
b_{+}^{q} & 0 \\
0 & 0
\end{array}\right]=\left[\begin{array}{lll}
u_{11} b_{+}^{q} u_{11}^{*} & u_{11} b_{+}^{q} u_{21}^{*} \\
u_{12} b_{+}^{q} u_{11}^{*} & u_{21} b_{+}^{q} u_{21}^{*}
\end{array}\right] .
$$

The (2,2)-entries in (3.4) show that $\left\langle b_{+}^{q} u_{21}^{*} \xi_{0}, u_{21}^{*} \xi_{0}\right\rangle=0$, for all $\xi_{0} \in \mathcal{H}_{0}$. Hence, $u_{21}^{*}$ maps $\mathcal{H}_{0}$ into ker $b_{+}^{q}=\{0\}$, implying that $u_{21}=0$. So, $u=u_{+} \oplus u_{0}$, where $u_{+}=u_{11}$ and $u_{0}=u_{22}$.

Now equating the $(1,1)$-entries in (3.4), we have $u_{+} b_{+}^{q} u_{+}^{*}=b_{+}^{q}$. Thus, $\overline{\operatorname{ran} u_{+}} \supseteq \overline{\operatorname{ran} b_{+}}=\mathcal{H}_{+}$, meaning that $u_{+}$has dense range. Furthermore, the condition $R\left[u^{*}\right] \geq R[b]$ implies that $R\left[u_{+}^{*}\right] \geq R\left[b_{+}\right]=1_{\mathcal{H}_{+}}$. Hence, $u_{+}^{*} u_{+}=1_{\mathcal{H}_{+}}$ and so $u_{+}$is a surjective isometry (i.e., a unitary). Therefore, $u_{+}$commutes with $b_{+}^{q}$. Passing to the $q$-th root, $u_{+}$commutes with $b_{+}$and therefore $u=u_{+} \oplus u_{0}$ commutes with $b=b_{+} \oplus 0$.

To prove that (2) implies (1), assume that $b^{q}=a^{p}, R\left[u^{*}\right] \geq R[b]$, and $u b=b u$. The matrix forms (3.2) of $b$ and $u$ satisfy $u b=b u$ only if $b_{+} u_{12}=0$ and $u_{21} b_{+}=0$. But ker $b_{+}=\{0\}$ shows that $b_{+} u_{12}=0$ is possible only if $u_{12}=0$. Likewise, passing to the adjoint of $u_{21} b_{+}=0$, the equation $b_{+} u_{21}^{*}=0$ holds only if $u_{21}^{*}=0$. Therefore, $u=u_{+} \oplus u_{0}$, where $u_{+}=u_{11}$ and $u_{0}=u_{22}$. 
As noted above, the assumption that $R\left[u^{*}\right] \geq R[b]$ implies $R\left[u_{+}^{*}\right] \geq R\left[b_{+}\right]$and, hence, $u_{+}$is an isometry. But moreover, $u b=b u$ implies that $u_{+} b_{+}=b_{+} u_{+}$, and so the range of the isometry $u_{+}$contains the closed range of $b_{+}$, namely $\mathcal{H}_{+}$. Thus, $u_{+}$is a unitary commuting with $b_{+}$and so $u_{+} b_{+}^{q} u_{+}^{*}=b_{+}^{q}$, which clearly implies that $u b^{q} u^{*}=b^{q}$. Now making use of $b^{q}=a^{p}$, we conclude that $u|a b| u^{*}=\frac{1}{p} a^{p}+\frac{1}{q} b^{q}$.

Now we extend Theorem 3.1 to arbitrary trace-class operators. To do so we shall make repeated use of the equation

$$
\left|a b^{*}\right|=v|| a|| b|| v^{*}
$$

where $b=v|b|$ is the polar decomposition of $b$.

Theorem 3.2. Let $a, b \in B(\mathcal{H})$ be trace-class operators, and suppose that $u \in$ $B(\mathcal{H})$ is a partial isometry. Let $p, q \in \mathbb{R}^{+}$such that $\frac{1}{p}+\frac{1}{q}=1$. Then the following statements are equivalent:

1. $u\left|a b^{*}\right| u^{*}=\frac{1}{p}|a|^{p}+\frac{1}{q}|b|^{q}$;

2. $|b|^{q}=|a|^{p}, R\left[u^{*}\right] \geq R[b]$, and $u b=v^{*}(b u) v$,

where $b=v|b|$ is the polar decomposition of $b$.

Proof. Assume first that $|b|^{q}=|a|^{p}, R\left[u^{*}\right] \geq R[b]$, and $u b=v^{*}(b u) v$. Note that $u v$ is a partial isometry. Indeed, since $v v^{*}=R[b]$, we have that $u^{*} u v=v$, or equivalently $v^{*} u^{*} u=v^{*}$. So $v^{*} u^{*} u v=v^{*} v$ is a projection, and this implies that $u v$ is a partial isometry.

The condition $u^{*} u b=b$ can be written as $u^{*} u v|b|=v|b|$. If we apply $v^{*}$ to this equality and take into account that $v^{*} v|b|=|b|$, then $(u v)^{*}(u v)|b|=|b|$. The condition $u b=v^{*} b u v$ can be written as $u v|b|=|b| u v$. So we have shown that the conditions (2) are equivalent to

$$
|b|^{q}=|a|^{p}, \quad R\left[(u v)^{*}\right] \geq R[|b|], \quad(u v) b=b(u v),
$$

with $u v$ a partial isometry. Then, from Theorem 3.1, we get that

$$
u v|| a|| b|| v^{*} u^{*}=\frac{1}{p}|a|^{p}+\frac{1}{q}|b|^{q},
$$

that is

$$
u\left|a b^{*}\right| u^{*}=\frac{1}{p}|a|^{p}+\frac{1}{q}|b|^{q} .
$$

Conversely, if we assume condition (1), we can write it as

$$
u v|| a|| b|| v^{*} u^{*}=\frac{1}{p}|a|^{p}+\frac{1}{q}|b|^{q},
$$


where $b=v|b|$ is the polar decomposition of $b$. From Theorem 2.2 applied to condition (1), we have that $|b|^{q}=|a|^{p}$, and $R\left[u^{*}\right] \geq R[b]$. Then again we know that $u^{*} u v=v$, and so $u v$ is a partial isometry, as we proved in the first paragraph. Now from equation (3.5) and Theorem 3.1, we have that $R\left[(u v)^{*}\right] \geq R[b]$ and $u v|b|=|b| u v$. As in the first part of the proof, these relations are easily seen to be equal to the ones in condition (2).

\section{Remarks and applications}

This work began with the goal of proving that equality in the operator Young inequality

$$
u\left|a b^{*}\right| u^{*} \leq \frac{1}{p}|a|^{p}+\frac{1}{q}|b|^{q},
$$

implies that $|a|^{p}=|b|^{q}$. While this problem remains open for arbitrary compact operators, it has been settled by Hirzallah and Kittaneh (in Remark 4, following Corollary 3 of $[\mathrm{HK}]$ ) for Hilbert-Schmidt operators by way of the remarkable inequality

$$
\left\|a b^{*}\right\|_{2}^{2}+\frac{1}{r^{2}}\left\||a|^{p}-|b|^{q}\right\|_{2}^{2} \leq\left\|\frac{1}{p}|a|^{p}+\frac{1}{q}|b|^{q}\right\|_{2}^{2},
$$

where $r=\max \{p, q\}$. Apart from the fact that the inequality itself is notable, there is something else that the inequality leads to: the surprising fact that to consider the case of equality in the operator inequality (4.1), one need only assume that there is equality in the Hilbert-Schmidt norm. Our results in the present paper are in the same vein: it is sufficient to assume only that $\operatorname{tr}\left(u\left|a b^{*}\right| u^{*}\right)=$ $\frac{1}{p} \operatorname{tr}\left(|a|^{p}\right)+\frac{1}{q} \operatorname{tr}\left(|b|^{q}\right)$.

Combining $[\mathrm{HK}]$ with Theorem 2.2, one has the following result, which shows the surprising "rigidity" of the Young inequality.

Theorem 4.1. Suppose $p, q \in \mathbb{R}^{+}$are such that $\frac{1}{p}+\frac{1}{q}=1$. If $a, b \in B(\mathcal{H})$ are trace-class operators and if $u \in B(\mathcal{H})$ is a partial isometry, then the following conditions are equivalent:

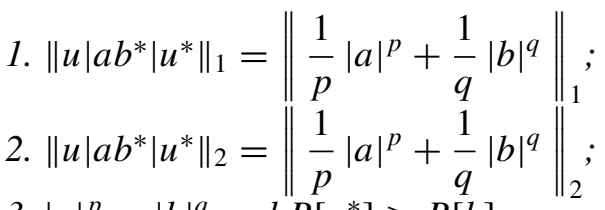

3. $|a|^{p}=|b|^{q}$ and $R\left[u^{*}\right] \geq R[b]$.

Proof. The only thing to be verified is that one can eliminate from consideration the partial isometry $u$ in (2). This can be done by using the arguments employed in (2.2) and (2.3), but applying them to the Hilbert-Schmidt norm instead of the 
trace. And then apply twice a reasoning like the one at the end of the proof of Theorem 2.2 to get that $R\left[u^{*}\right] \geq R[b]$.

The rigidity condition mentioned in the Theorem 4.1 is in fact stronger than the way it was stated. In particular, for matrices a rather complete statement can be established:

Theorem 4.2. Suppose $p, q \in \mathbb{R}^{+}$are such that $\frac{1}{p}+\frac{1}{q}=1$. If $a, b \in M_{n}(\mathbb{C})$ are matrices and if $u \in M_{n}(\mathbb{C})$ is a partial isometry, then the following conditions are equivalent:

1. $\left\||u| a b^{*}\left|u^{*}\right|\right\|=\left\|\left.\left|\frac{1}{p}\right| a\right|^{p}+\frac{1}{q}|b|^{q} \mid\right\|$ for every unitarily invariant norm ||$|\cdot|||$

2. $|a|^{p}=|b|^{q}$ and $R\left[u^{*}\right] \geq R[b]$.

Proof. The assertion (1) implies (2) is clear from Theorem 4.1, for the trace norm is a unitarily invariant norm.

Conversely, if $|a|^{p}=|b|^{q}$, then $s_{j}(a)^{p}=s_{j}(b)^{q}$ for every $j=1, \ldots, n$, where $s_{j}$ denotes the $j$-th singluar value. We write $b=v|b|$ the polar decomposition of $b$ and note, as in the proof of Theorem 3.2, that $u^{*} u v=v$ and $v^{*} v|b|=|b|$. Then, writing $\|\cdot\|_{(k)}$ for the $k$-th Ky Fan norm, we have (using that $s_{j}\left(x^{*} x\right)=s_{j}\left(x x^{*}\right)$ for every $j$ )

$$
\begin{aligned}
\left\|u\left|a b^{*}\right| u^{*}\right\|_{(k)} & =\sum_{j=1}^{k} s_{j}\left(u\left|a b^{*}\right| u^{*}\right)=\sum_{j=1}^{k} s_{j}\left(u v|b|^{q} v^{*} u^{*}\right) \\
& =\sum_{j=1}^{k} s_{j}\left(|b|^{q / 2} v^{*} u^{*} u v|b|^{q / 2}\right)=\sum_{j=1}^{k} s_{j}\left(|b|^{q / 2} v^{*} v|b|^{q / 2}\right) \\
& =\sum_{j=1}^{k} s_{j}\left(|b|^{q}\right)=\sum_{j=1}^{k} s_{j}\left(\frac{1}{p}|a|^{p}+\frac{1}{q}|b|^{q}\right) \\
& =\left\|\frac{1}{p}|a|^{p}+\frac{1}{q}|b|^{q}\right\|_{(k)} .
\end{aligned}
$$

Thus, we have equality for all the Ky Fan norms, and so we have equality for every unitarily invariant norm (see Theorem IV.2.2 of [Ba]).

Let us also mention the fact that one cannot expect in general that equality in just one unitarily invariant norm forces condition (2) in Theorem 4.2. For instance one can consider the Ky Fan norm $\|\cdot\|_{(1)}$ (that is, the operator norm). Let

$$
a=\left[\begin{array}{ll}
1 & 0 \\
0 & \epsilon
\end{array}\right], \quad b=\left[\begin{array}{ll}
1 & 0 \\
0 & 0
\end{array}\right] .
$$


Then clearly

$$
\||a b|\|=1, \quad\left\|\frac{1}{p} a^{p}+\frac{1}{q} b^{q}\right\|=1,
$$

but $a^{p} \neq b^{q}$. With the same idea it is easy to build examples for all the $k$-th Ky Fan norms, $k<n$. (However, the case $k=n$ is the trace norm.)

While working on this paper, the authors frequently encountered problems concerning where the involution $*$ ought to be placed in conditions like $R\left[u^{*}\right] \geq$ $R[b]$. This difficulty is an illustration of an insightful and eloquent comment of R.C. Thompson (at the bottom of page 87 of [T]) on the challenges one faces in formulating operator inequalities. With Young's inequality, at least for positive operators, in the end it so happens that the concern about where to place the $*$ really does not matter. Indeed, this is because the condition of equality is so strong: if we have trace-class operators $a, b \geq 0$ and a partial isometry $u$ such that

$$
u|a b| u^{*}=\frac{1}{p} a^{p}+\frac{1}{q} b^{q},
$$

then by Theorem 3.1 we know that $a^{p}=b^{q}, R\left[u^{*}\right] \geq R[b]$ and $u b=b u$. We have that

$$
u b^{q} u^{*}=b^{q} .
$$

But, since $u^{*} u b=b$, if we conjugate the above equation with $u^{*}$ and $u$, we get

$$
b^{q}=u^{*} b^{q} u
$$

which implies that

$$
u^{*}|a b| u=\frac{1}{p} a^{p}+\frac{1}{q} b^{q} .
$$

That is, in the operator equality the roles of $u$ and $u^{*}$ can be interchanged.

Acknowledgements. This research was supported in part by a grant from the Natural Sciences and Engineering Research Council of Canada. The authors wish to thank Juliana Erlijman and Renying Zeng for discussions regarding the material herein. The first named author also thanks D. Farenick and J. Erlijman for their warm hospitality during his stay at the University of Regina, where this work began.

\section{References}

[A] Ando, T.: Matrix Young Inequalities. Oper. Theory Adv. Appl 75, 33-38 (1995)

[Ba] Bathia, R.: Matrix Analysis, Springer-Verlag, New York, 1997

[BK] Bathia, R., Kittaneh, F.: On the singular values of a product of operators, SIAM J. Matrix Anal. Appl. 11, 272-277 (1990)

[EFZ] Erlijman, J., Farenick, D.R., Zeng, R.: Young's inequality in compact operators. Oper. Theory Adv. Appl. 130, 171-184 (2001)

[GK] Gohberg, I.C., Krein, M.G.: Introduction to the Theory of Linear Nonselfadjoint Operators, American Mathematical Society, Providence, 1969 
[HK] Hirzallah, O., Kittaneh, F.: Matrix Young inequalities for the Hilbert-Schmidt norm, Linear Algebra Appl. 308, 77-84 (2000)

[T] Thompson, R.C.: The matrix-valued triangle inequality: quaternion version. Linear Multilinear Algebra 25, 85-91 (1989)

[Z] Zeng, R.: Young's Inequality in Compact Operators, Ph.D. Dissertation, University of Regina, 2002 